\title{
Synthesis and Integration of Poly(1-Vinylimidazole) Polymer \\ Electrolyte in Dye Sensitized Solar Cells by \\ Initiated Chemical Vapor Deposition
}

Austin G. Kuba*1, Yuriy Y. Smolin*1, Masoud Soroush ${ }^{1}$, Kenneth K. S. Lau ${ }^{\dagger 1}$

\begin{abstract}
Initiated chemical vapor deposition (iCVD) is used to integrate poly(1-vinylimidazole) (PVIZ) into the mesoporous photoanode of dye sensitized solar cells (DSSCs). To our knowledge, this is the first reported demonstration of PVIZ via iCVD as a novel polymer electrolyte in DSSCs. Fourier transform infrared (FTIR) spectroscopy and X-ray photoelectron spectroscopy (XPS) verified the iCVD polymer as stoichiometric PVIZ. Polymer deposition rate was found to increase with increasing reactor pressure and decreasing substrate temperature. The kinetic studies further determined that the surface polymerization reaction is first order with respect to the surface monomer concentration. The power conversion efficiency of DSSCs incorporating crosslinked PVIZ was found to be $27 \%$ higher than that of liquid electrolyte DSSCs as a result of higher open circuit voltage and short circuit current.
\end{abstract}

Keywords: initiated chemical vapor deposition (iCVD); poly(1-vinylimidazole); kinetic analysis; polymer electrolyte; dye sensitized solar cell; pore penetration

\footnotetext{
${ }^{1}$ Department of Chemical and Biological Engineering, Drexel University, 3141 Chestnut Street, Philadelphia, PA 19104, U.S.A.

* Authors contributed equally to the work.

† Corresponding author: klau@drexel.edu
} 


\section{Introduction}

The dye sensitized solar cell (DSSC), first introduced by the Grätzel group in $1991^{1}$, is a promising photovoltaic technology that has demonstrated cell efficiencies of over $14 \%^{2}$ and can be manufactured at a lower cost than conventional silicon-based homojunction solar cells ${ }^{3}$. Most DSSCs are composed of a mesoporous $\mathrm{TiO}_{2}$ photoanode coated on a transparent conducting oxide (TCO) layer ${ }^{4}$. This $\mathrm{TiO}_{2}$ photoanode is sensitized with a monolayer of a ruthenium dye like di-tetrabutylammonium cisbis(isothiocyanato)bis(2,2'-bipyridyl-4,4'-dicarboxylato)ruthenium(II), commonly known as N719. In a liquid electrolyte DSSC, sandwiched between the photoanode and the platinized TCO cathode is a liquid electrolyte containing an iodide/triiodide redox couple. The dye is photoexcited by incident light and injects electrons into the mesoporous $\mathrm{TiO}_{2}$. The electrons then move via diffusion to the transparent conducting oxide, go through an external load, and finally come back to the platinized cathode. The iodide/triiodide redox couple then accepts the electrons from the cathode and transports them back to the photoanode to regenerate the dye.

A major limitation of the current DSSC design is the liquid electrolyte, which is prone to leakage and evaporation along with being corrosive to metal contacts such as silver ${ }^{5,6}$. Furthermore, electron recombination loss is significant at the $\mathrm{TiO}_{2}$-electrolyte interface. Therefore, replacing the liquid electrolyte can enhance DSSC performance and address the other major issues with liquid electrolytes. In our previous work, it was shown that replacing the liquid electrolyte with a polymer electrolyte leads to higher performance along with the ability to tune the current-voltage $(J-V)$ behavior of the cell $^{6,7}$. To a major extent, this increase has been attributed to a stabilized and enhanced 
photoanode-electrolyte interface. Furthermore, polymer electrolytes have relatively high ionic conductivity, which make them an excellent alternative to the liquid electrolyte ${ }^{8}$.

To ensure optimal performing DSSCs, there needs to be intimate contact between the polymer electrolyte and the $\mathrm{TiO}_{2}$ throughout the entire photoanode layer to ensure the redox couple can reach the absorbed dye. However, due to the nanostructured, mesoporous nature of the $\mathrm{TiO}_{2}$ photoanode and the effects of viscosity on fluid transport in such a structure, conventional polymer deposition techniques such as spin casting and dip coating lead to ineffective pore penetration. Often only the top 2 $\mu \mathrm{m}$ out of a $10 \mu \mathrm{m}$ photoanode layer is in contact with the polymer with the remaining 8 $\mu \mathrm{m}$ below completely void of polymer ${ }^{9-12}$. This reduces device performance because a large portion of the adsorbed dye is not accessible and cannot be regenerated. To overcome the limitation of the methods for incorporating polymer electrolytes into the mesoporous $\mathrm{TiO}_{2}$, we employ a novel polymerization technique called initiated chemical vapor deposition (iCVD). iCVD is a solvent-free polymerization method that relies on gas-to-surface reactions at low pressures. This makes it especially useful for cases that require penetration of sub-micron and nanometer-sized pores. Another advantage of this method of deposition for DSSCs is that it does not require the use of any liquid solvents, which may get trapped in the photoanode and degrade performance since they are difficult to remove ${ }^{7}$.

In iCVD, the monomer and initiator are delivered into a vacuum chamber as vapors (Fig. 1). They flow past a series of heated filaments whereupon the initiator is thermally decomposed into free radicals without degrading the monomer. Subsequently, monomer vapor adsorbs on a cooled substrate surface and surface polymerization 
occurs through monomer addition and polymer chain propagation at activated initiator sites. Utilizing this approach, the entire photoanode thickness can be covered with polymer very efficiently ${ }^{5,7}$. Compared with liquid electrolyte additives, the effect of chemical groups of the polymer electrolytes is not as well studied, although recent work has provided some insight into electron transfer kinetics and possible reasons for cell efficiency improvements when comparing polymers and liquid additives ${ }^{13-16}$. Our previous work on polymer electrolytes deposited via iCVD has shown that these electrolytes can reduce charge recombination loss at the $\mathrm{TiO}_{2}$-electrolyte interface in DSSCs and negatively shift the $\mathrm{TiO}_{2}$ conduction band to improve the open circuit voltage of DSSCs ${ }^{5-7}$.

Here, iCVD is utilized for the synthesis and integration of poly(1-vinylimidazole) (PVIZ) in DSSCs to investigate the impact of PVIZ on DSSC performance. PVIZ is potentially a more viable polymer electrolyte for use in DSSCs than poly(4vinylpydridine) used in our previous work ${ }^{6}$. The work showed that a polymer electrolyte with more basic character yields higher DSSC short circuit current as a result of strong acid-base interactions with the $\mathrm{TiO}_{2}$ electrode that creates a blocking layer and reduces electron recombination at the interface. Furthermore, the work showed that a polymer which binds strongly to metal ions such as $\mathrm{Li}^{+}$(counterion of iodide in DSSCs) is able to improve DSSC open circuit voltage by reducing the surface $\mathrm{Li}^{+}$concentration on $\mathrm{TiO}_{2}$ and shifting the $\mathrm{TiO}_{2}$ conduction band to a more negative potential. The hypothesis here is that a polymer electrolyte that is strong in both characteristics, such as PVIZ, would be able to enhance both photocurrent and photovoltage, and lead to more enhanced DSSCs. Reports on the use of polyionic liquids in DSSCs have shown that imidazolium- 
based polyionic liquids consistently outperform pyridinium-based ones ${ }^{17}$. In direct methanol fuel cells, PVIZ has been applied as a copolymer for proton conduction ${ }^{18}$, and PVIZ has been used in the form of a hydrogel to act as a mercury ion chelating agent for fixing the metal cation ${ }^{19}$. It is therefore conceivable that PVIZ would favor ion conduction of the iodide-triiodide redox couple. In this work, PVIZ is conformally coated onto the high surface area, mesoporous $\mathrm{TiO}_{2}$ photoanode. To understand more carefully the role of the polymer at the photoanode-electrolyte interface, only a thin conformal coating wrapping the $\mathrm{TiO}_{2}$ nanoparticles is applied while the remaining bulk of the electrolyte is acetonitrile. To ensure conformal coating within highly porous networks, it is important to control the balance between transport and reaction dynamics. Ideally, iCVD must be performed under reaction-limited conditions where mass transport by Knudsen and surface diffusion is rapid ${ }^{7}$. The key iCVD parameter that affects such dynamics is the fractional saturation ratio of the monomer, defined as the $Z$ ratio of monomer partial pressure in the gas phase to its saturation pressure at the temperature of the substrate surface ${ }^{20,21}$. This study therefore first aims to study the kinetic behavior of the iCVD synthesis of PVIZ. The second part of this study aims to elucidate the DSSC behavior with PVIZ at the electrode interface.

\section{Materials and Methods}

\section{1. iCVD of Poly(1-Vinylimidazole)}

1-Vinylimidazole monomer (VIZ; >99\% Sigma Aldrich) and di-tert-butyl-peroxide initiator (TBPO; 98\% Sigma Aldrich) were used as purchased without further purification. Figure

2 shows the reaction of $V I Z$ with TBPO to form PVIZ through free-radical addition 
polymerization. $\mathrm{VIZ}$ was heated to $55{ }^{\circ} \mathrm{C}$ to ensure sufficient vapor flowrate and TBPO was kept at $20^{\circ} \mathrm{C}$. The flowrates of the monomer and initiator into the reactor were held constant at 0.5 and $0.1 \mathrm{sccm}$ (standard cubic $\mathrm{cm}$ per min), respectively. Chromalloy-O (Goodfellow) was used as the filament material and a Sorensen DLM 60-10DC power supply was used to resistively heat the filament wires to $280{ }^{\circ} \mathrm{C}$ by passing $1.25 \mathrm{~A}$ current and $20.7 \mathrm{~V}$ voltage. The deposition substrate was placed on a cooling stage below the array of filament wires and cooled by backside contact with a thermal fluid flowing through a Thermo Scientific RTE-7 chiller.

To study the effect of processing conditions on deposition kinetics, two series of iCVD reactions were performed. In the first series the substrate temperature was held constant at $18{ }^{\circ} \mathrm{C}$, while the pressure in the reactor was varied from 0.15 to 0.25 Torr in steps of 0.05 Torr. In the second series, the reactor pressure was held constant at 0.25 Torr, while the substrate temperature to was set to $17,18.5,28,35$, and $41^{\circ} \mathrm{C}$. The fractional monomer saturation ratio, i.e. the ratio of monomer partial pressure in the reactor over the saturation pressure at the substrate's surface $\left(Z=P_{m} / P_{m}^{s a t}\right)$, is well correlated with the rate of the deposition, because it is a good indicator of the surface availability or concentration of the monomer (e.g. when $Z=1$, monomer is fully saturating the surface and at its condensation point). The partial pressure of the monomer in the reactor was controlled via reactor pressure as the mole fraction of monomer in the gas phase was constant at 0.83 . Saturation pressure at the surface of the substrate was controlled by the substrate temperature and predicted using the Clausius-Clapeyron equation based on known data points on the vapor pressure of VIZ. 


\subsection{Polymer Characterization}

Fourier transform infrared (FTIR) spectroscopy was performed on a Thermo Nicolet 6700 spectrometer in normal transmission mode. Before all scans, the chamber was purged for at least 2 min with nitrogen gas to reduce carbon dioxide and water vapor in the chamber. A background spectrum of bare silicon was taken from a wavenumber range of $400-4000 \mathrm{~cm}^{-1}$ with 128 scans. All scans were performed using a deuterated triglycine sulfate (DTGS) detector. Once the background sample was taken and removed, the iCVD PVIZ sample deposited on a silicon substrate was placed in the spectrometer, purged again with nitrogen gas for at least 2 min, and scanned 128 times against the bare silicon background. The spectra were baseline corrected and then compared with literature data to identify the characteristic peaks for PVIZ. FTIR was also carried out with the same instrument and acquisition parameters on the VIZ monomer and a commercially available PVIZ polymer (Polymer Source). VIZ liquid was cast directly onto a silicon substrate while the commercial PVIZ was dissolved in ethanol, sonicated for $30 \mathrm{~min}$, cast from solution onto a silicon substrate, and subsequently dried for 30 min in a vacuum oven.

X-ray photoelectron spectroscopy (XPS) was performed using a Physical Electronics VersaProbe 5000 with a scanning monochromatic source from an Al anode and with dual beam charge neutralization. High resolution XPS spectra of $\mathrm{C} 1 \mathrm{~s}$, O1s, and $\mathrm{N} 1 \mathrm{~s}$ were acquired at $25 \mathrm{~W}$ with a $100 \mu \mathrm{m}$ spot size using an energy step size of 
$0.05 \mathrm{eV}$, a pass energy of 23.50 , and a dwell time of $50 \mathrm{~ms}$. The nitrogen signal was taken with 768 scans while the carbon and oxygen signals consisted of 192 scans each.

Gel permeation chromatography was carried out on a Shimadzu LC-20AD HPLC with two Agilent PLgel MIXED-B columns in series and a Shimadzu RID-20A refractive index detector to determine a representative molecular weight of iCVD PVIZ (from the pressure series run at 0.25 Torr). N,N-Dimethylacetamide (DMAc; $>99.9 \%$ Sigma Aldrich) with $0.05 \mathrm{M} \mathrm{LiCl}$, as the elution medium, flowed at a rate of $1 \mathrm{ml} / \mathrm{min}$ through the columns maintained at $55^{\circ} \mathrm{C}$. The instrument was calibrated with 10 different PMMA molecular weight standards. Both iCVD and commercial PVIZ, made to a DMAc solution of $2 \mathrm{mg} / \mathrm{ml}$, were analyzed.

The thickness of each PVIZ film was estimated using an M-2000U variable angle spectroscopic ellipsometer (VASE) from J.A. Woollam with scans at angles of 60, 65, 70,75 , and 80 degrees. Raw amplitude and phase change data were fitted to an optical film model created in the WVASE32 software that consisted of a bare silicon substrate, a $6 \mathrm{~nm}$ native oxide layer, and a Cauchy model layer to represent the PVIZ polymer film. By minimizing the mean squared error, estimates of film thickness and optical constants were calculated using the converged model. The calculated film thickness was then divided by the total reaction time to obtain an average deposition rate for each iCVD run.

\subsection{DSSC Fabrication}

Fluorine doped tin oxide glass (FTO; Hartford Glass, TEC7) was cleaned via sonication at $40{ }^{\circ} \mathrm{C}$ for 15 min each in dilute soap solution, 50:50 acetone-ethanol mixture, and 
isopropanol. Next, $\mathrm{TiO}_{2}$ paste was prepared from $\mathrm{P} 25 \mathrm{TiO}_{2}$ nanoparticles (Evonik) as previously described ${ }^{6,22}$. The $\mathrm{TiO}_{2}$ paste was doctor-bladed onto the cleaned and dried FTO glass. The prepared substrate was heated step-wise from 50 to $500{ }^{\circ} \mathrm{C}$ in $10^{\circ} \mathrm{C}$ increments. The sample was held at each $10^{\circ} \mathrm{C}$ step for 2 min and finally at $500{ }^{\circ} \mathrm{C}$ for $30 \mathrm{~min}$. The hotplate was then set to $80^{\circ} \mathrm{C}$, allowing the substrate to cool. Once the photoanode cooled to $80{ }^{\circ} \mathrm{C}$, it was immersed in a $3 \times 10^{-4} \mathrm{M}$ solution of $\mathrm{N} 719$ dye (Solaronix) in pure ethanol for at least $24 \mathrm{~h}$. The photoanode was then rinsed with ethanol, dried, and then immediately placed in the iCVD reactor. Since PVIZ was soluble in acetonitrile, the PVIZ needed to be crosslinked during iCVD synthesis to ensure material stability. Ethylene glycol diacrylate (EGDA) was used as the polymer crosslinker. The flowrates of TBPO, VIZ, and EGDA were maintained at 1.0, 1.5, and $1.1 \mathrm{sccm}$, respectively. The reactor pressure was 0.25 Torr and the stage temperature was $29^{\circ} \mathrm{C}$. This resulted in a $Z$ value of 0.11 for the $\mathrm{VIZ}$ monomer. This $Z$ value was selected after several preliminary runs were made at different $Z$ s that indicated these conditions resulted in a reaction-limited regime for good conformal coating of the mesoporous $\mathrm{TiO}_{2}$ photoanode. The reaction was allowed to proceed for $1 \mathrm{~h}$, which was deemed a sufficient run time to coat the inner pore surfaces of the photoanode. After the reaction was complete, the polymer electrolyte photoanode was soaked for $6 \mathrm{~h}$ at room temperature in acetonitrile containing $0.05 \mathrm{M}$ lithium iodide (Aldrich 99.9\%) and $0.5 \mathrm{M}$ iodine (Aldrich 99.9\%) to incorporate the redox couple in the PVIZ and fill the remaining space with electrolyte. For comparison, an uncoated photoanode was used with the same acetonitrile liquid electrolyte. A platinized cathode was prepared by cleaning another FTO glass as described above, and then placing $50 \mathrm{ml}$ of $5 \mathrm{mM}$ 
chloroplatinic acid hydrate (Aldrich, 99.9\%) in 2-propanol (Aldrich, 99\%) and annealing it at $500{ }^{\circ} \mathrm{C}$ for $40 \mathrm{~min}$. The electrolyte-filled photoanode was contacted with the platinized cathode and held together with fastening clips. A tape was used as a spacer support between the two electrodes to prevent electrical shorts.

\subsection{DSSC Evaluation}

Current-voltage characteristics of the DSSCs were measured using a Gamry Reference 600 potentiostat under simulated solar illumination. The light source was a $300 \mathrm{~W}$ Xe lamp calibrated to the AM 1.5 spectrum at $100 \mathrm{~mW} / \mathrm{cm}^{2}$ intensity using neutral density filters. Linear sweep voltammetry was initiated at open circuit followed by an applied bias voltage up to $700 \mathrm{mV}$ at a scan rate of $25 \mathrm{mV} / \mathrm{s}$ and a step size of $2 \mathrm{mV}$. An equilibration time of $5 \mathrm{~s}$ was given before any measurements were taken.

\section{Results and Discussion}

\subsection{PVIZ Structure and Composition}

The Fourier transform infrared (FTIR) spectra of VIZ monomer, iCVD PVIZ polymer, and commercial PVIZ are shown in Fig. 3. Peak positions and assignments are listed in Table 1. The spectral peaks for all PVIZ polymers from the various iCVD runs in the two deposition series were identical and compare well to literature values reported by Talu et al. $^{23}$ and Lippert et al. ${ }^{24}$ for PVIZ synthesized in the liquid phase via free radical polymerization. In addition, the spectra of the ICVD and commercial PVIZ are nearly identical, further lending support that PVIZ produced via iCVD resembles stoichiometric PVIZ polymer. The peaks at 1497, 1284, 1083, 744 and $663 \mathrm{~cm}^{-1}$ are all attributed to 
various vibrational modes of the imidazole ring, see Table 1. The peak at $3108 \mathrm{~cm}^{-1}$, attributed to the $\mathrm{C}-\mathrm{H}$ stretch on the imidazole ring, is stronger in relative magnitude for $\mathrm{PVIZ}$ than VIZ but is present in both. The presence of these peaks in both $\mathrm{VIZ}$ and $\mathrm{PVIZ}$ vibrational spectra supports the assertion that the imidazole ring is unbroken in the polymerization and was not degraded even in the presence of the heated filament. This highlights one of the key strengths of the iCVD approach in that the technique is gentle enough to preserve the chemical functionality of the monomer as has been demonstrated for a variety of different polymer systems ${ }^{25}$. The main difference between the VIZ and PVIZ spectra is the disappearance of the sharp $1647 \mathrm{~cm}^{-1}$ peak in the latter, which is attributed to the loss of the $\mathrm{C}=\mathrm{C}$ vinyl bond. This confirms that the monomer has polymerized cleanly through the free radical addition of the vinyl bond. In addition, both iCVD and commercial PVIZ have peaks in the range of 2935-2965 $\mathrm{cm}^{-1}$ representing $\mathrm{CH}$ and $\mathrm{CH}_{2}$ stretches on the polymer backbone that are absent in the $\mathrm{VIZ}$ spectrum $^{24}$. The presence of backbone chain stretching in the PVIZ polymer again supports the formation of long chain polymer. More quantitatively, GPC was performed to determine polymer molecular weight and polydispersity index (PDI), which gave the iCVD PVIZ a representative $M_{\mathrm{n}}$ and PDI of $5,860 \mathrm{~g} / \mathrm{mol}$ and 1.62 , respectively, while that for the commercial PVIZ had values of $12,950 \mathrm{~g} / \mathrm{mol}$ and 1.63 (see Figure S1 in Supporting Information).

X-ray photoelectron spectroscopy (XPS) was used to quantitatively determine the chemical composition of PVIZ. From Fig. 4a, the high resolution N1s spectrum can be fitted to two separate peaks, one at $398.6 \mathrm{eV}$ corresponding to the amine group $(-\mathrm{N}<)$ and one at $400.5 \mathrm{eV}$ attributed to the imine group $(-\mathrm{N}=)^{26,27}$. From the ratio of the 
intensities of the two peaks, the iCVD PVIZ yields a value of $0.93: 1$ amine to imine, which is very close to the ideal ratio of $1: 1$ based on the chemical stoichiometry of PVIZ (see Table 2). From Fig. 4b, the high resolution $\mathrm{C} 1 \mathrm{~s}$ spectrum can be fitted to three separate peaks centered at $284.5,285.3$, and $286.3 \mathrm{eV}$, corresponding to $\mathrm{C}-\mathrm{H}, \mathrm{C}-\mathrm{N}$, and $\mathrm{N}=\mathrm{C}-\mathrm{N}$ carbon bonding environments, respectively ${ }^{26}$. As seen in Table 2, based on the relative intensities of the fitted peaks, the iCVD PVIZ ratio of 5.0:2.9:1.2 is close to the theoretical ratio of 5:3:1 for stoichiometric PVIZ. XPS was also done on commercial PVIZ, which had nearly identical peak locations and relative intensities to iCVD PVIZ (see Figure S2 and Table S1 in Supporting Information). The XPS data provides quantitative evidence, in addition to the qualitative FTIR data, that PVIZ has been synthesized cleanly by iCVD and is spectroscopically identical to prior literature studies

on PVIZ synthesized in the liquid phase ${ }^{23,24,26}$. XPS did detect a very small amount of oxygen species on the surfaces of both iCVD and commercial PVIZ (see Figure S3 and Table S2 in Supporting Information), which can be attributed to minute quantities of surface adsorbed contaminants.

\subsection{Reaction Kinetics}

To study the effect of various conditions on the deposition rate of PVIZ, two series of films were deposited on silicon substrates. The first series held substrate temperature constant and varied reactor pressure while the second series held pressure constant and varied substrate temperature. Variable angle spectroscopic ellipsometry (VASE) was used to estimate film thickness through a Cauchy model of the polymer. Deposition rate was then derived by dividing the film thickness with the deposition run time. With 
the pressure series, an increase in pressure resulted in an increase in deposition rate. With the substrate temperature series, a decrease in temperature yielded to an increase in deposition rate. This unusual phenomena can be explained by the iCVD process being adsorption-limited rather than intrinsic reaction-limited ${ }^{20,21}$, so a cooler substrate promotes greater monomer adsorption and leads to faster polymer film deposition.

Figure 5 shows the trend of deposition rate for all the iCVD runs from the two series plotted as a function of $Z$, the fractional saturation ratio of the monomer, defined as the ratio of the monomer partial pressure in the gas phase to its saturation pressure at the substrate temperature. For both deposition series, the data collapses into a single linear trend $\left(R^{2}=0.95\right)$ within the range of iCVD conditions explored and agrees with previous studies on iCVD kinetics for other polymer systems ${ }^{20,21}$. The deposition rate is equivalent to the polymer growth rate, while the $Z$ ratio represents the monomer availability or monomer concentration at the surface. This linear behavior suggests that polymerization kinetics is first order with respect to monomer concentration, which is consistent with a thermally initiated free radical polymerization mechanism for vinyl polymer systems ${ }^{28}$.

\subsection{DSSC Performance}

PVIZ was found to be soluble in standard DSSC liquid electrolytes. To be used in a dye sensitized solar cell (DSSC), which utilizes acetonitrile as the bulk electrolyte solvent, PVIZ must be chemically crosslinked to prevent dissolution during DSSC fabrication as the redox couple in acetonitrile was added after layering the PVIZ within the photoanode. As previous work showed the successful use of EGDA to crosslink other 
vinyl polymers by $\mathrm{iCVD}^{29,30}$, EGDA was used here to crosslink PVIZ. A proper ratio of the $Z$ values of the crosslinker and monomer leads to an insoluble polymer film; i.e., there needs to be a sufficient amount of crosslinker during the polymer growth process to produce enough crosslinks. For $\mathrm{PVIZ}$, we found that a ratio of $Z_{\text {xlinker }} / Z_{\text {monomer }}$ of 4 resulted in an insoluble film without incorporating too much crosslinker that might influence the PVIZ benefits that we are aiming for in the DSSCs. Figure 6 shows the resulting current density-voltage $(J-V)$ characteristics of $P V I Z$ and liquid electrolyte DSSCs under 1 sun filtered to $1.5 \mathrm{AM}$. The short circuit current density $\left(J_{\mathrm{sc}}\right)$ with and without PVIZ is 5 and $3.97 \mathrm{~mA} / \mathrm{cm}^{2}$, respectively, while the open circuit voltage $\left(V_{o c}\right)$ with and without PVIZ is 380 and $365 \mathrm{mV}$, respectively. The fill factor with and without PVIZ cell is 42 and $43 \%$, respectively. These correspond to cell efficiencies of 0.80 and $0.63 \%$ with and without PVIZ. The slight decrease in the fill factor can be attributed to a slightly greater charge transfer resistance caused by the thin polymer coating on the $\mathrm{TiO}_{2}$ photoanode.

The increase in $J_{\text {sc }}$ and $V_{\text {oc }}$ with PVIZ integrated into the DSSC photoanode can be attributed to the effect of the polymer and specifically its chemical functionality on interfacial processes at the electrode-electrolyte interface. Figure 6 clearly shows that the polymer coating improves the short circuit current. Previous work on liquid electrolyte DSSCs revealed that additives, especially nitrogen containing molecules, can play a key role in enhancing DSSC performance due to physical and chemical properties of the nitrogen-containing compounds such as the partial charge, dipole moment and ionization energy ${ }^{31}$. Polymers and additives with a more basic nature can form a 'blocking layer' on the $\mathrm{TiO}_{2}$ surface due to Lewis acid-base interactions. Although 
a strong base is not a necessary requirement for a DSSC polymer-electrolyte, our previous work has shown that basic polymer electrolytes can dramatically reduce the electron recombination rate constant while an acidic one does not ${ }^{6}$. Furthermore, previous experimental reports with introducing 4-tert-butylpyridine (TBP) as a electrolyte additive has shown a reduction in the recombination rate constant by 1-2 orders of magnitude ${ }^{16,32}$. This is believed to enhance the short circuit current by blocking the invasion of triiodide onto the $\mathrm{TiO}_{2}$ surface as basic compounds have an affinity to adsorb onto the acidic $\mathrm{TiO}_{2}$ surface ${ }^{33}$. Furthermore, our previous work ${ }^{6}$ indicated that poly(4-vinylpyridine) (P4VP) can act as blocking layer due to the Lewis acid-base interaction of the nitrogen-containing group in the polymer with the $\mathrm{TiO}_{2}$ photoanode. This blocking layer reduces the electron recombination rate and increases short circuit current density $\left(J_{s c}\right)$. The imidazole group is 60 times more basic than the pyridine group based on their respective pKb values. This suggests that PVIZ should form a strong blocking layer compared to P4VP, which helps to reduce back electron transfer between the $\mathrm{TiO}_{2}$ surface and triiodide in the electrolyte. We believe this causes the higher current density in our PVIZ integrated DSSCs.

In DSSCs, the open circuit voltage is directly related to the fermi energy of the $\mathrm{TiO}_{2}$ surface, which is in turn influenced by the surface charge of the $\mathrm{TiO}_{2}$. Previous work from multiple groups showed that the coordination and formation of complexes between lithium iodide salt and DSSC additives hinder $\mathrm{Li}^{+}$from adsorbing onto the $\mathrm{TiO}_{2}$ photoanode ${ }^{34,35}$. This alters the $\mathrm{TiO}_{2}$ surface charge and shifts the fermi energy level. Specifically, as illustrated by the cation-promoted electron injection model, the conduction band of $\mathrm{TiO}_{2}$ shifts positively with increasing surface $\mathrm{Li}^{+}$concentrations ${ }^{34}$, 
and as the conduction band shifts positively, the $V_{o c}$ in the cell is reduced. Our previous work on polymer electrolytes suggested that the polymer electrolytes can form complexes with lithium, leading to a negative shift in the $\mathrm{TiO}_{2}$ conduction band and an increase in the open circuit voltage ${ }^{5-7}$. It has been shown that PVIZ can coordinate strongly with metal ions, and therefore it has the potential to bind well to lithium ions present in the DSSC electrolyte ${ }^{36,37}$. This is a possible explanation for why the open circuit voltage is higher in the PVIZ integrated DSSC. It is also possible that nitrogen from the $\mathrm{PVIZ}$ causes a negative shift in the conduction band of $\mathrm{TiO}_{2}$ itself, increasing $V_{\text {oc. }}$ This was observed for heterocyclic nitrogen-containing additives where, as the partial charge of nitrogen was decreased on the heterocyclic ring, $V_{o c}$ increased $^{31}$. Most likely, a combination of both factors contribute to the increase in the open circuit voltage.

It should be noted that the cell efficiencies here, even for the liquid electrolyte DSSC, are lower than those for state-of-the-art DSSCs, which indicate our systems are not fully optimized. For this work, we have focused on demonstrating an initial proof-ofconcept towards the integration of PVIZ into DSSCs and to investigate any improvements in performance the PVIZ might potentially offer without considering other device enhancements, such as adding an anode blocking layer, a light scattering layer, and cleaning the $\mathrm{TiO}_{2}$ surface with a $\mathrm{TiCl}_{4}$ treatment. This initial DSSC study, which showed increases in both $J_{s c}$ and $V_{o c}$, is significant as previous studies on polymer electrolytes and other additives typically only offer enhancements in either $J_{\mathrm{sc}}$ or $V_{\text {oc }}$, but not in both. Our future DSSC work will focus on optimizing device architecture and components, such as optimizing the $\mathrm{TiO}_{2}$ layer thickness, optimizing the amount of $\mathrm{PVIZ}$ 
incorporated, adding a compact $\mathrm{TiO}_{2}$ layer between the $\mathrm{FTO}$ and the mesoporous $\mathrm{TiO}_{2}$ photoanode to reduce back electron transfer that occurs on the $\mathrm{FTO}^{38}$, adding a scattering layer of $\mathrm{TiO}_{2}$ to increase light harvesting ${ }^{39}$, and adding a $\mathrm{TiCl}_{4}$ post treatment on the $\mathrm{TiO}_{2}$ layer, which is known to improve photocurrent ${ }^{40}$. Here, we chose not to include these enhancements but to focus on isolating the effects of only the polymer on $J-V$ behavior.

\section{Conclusions}

Poly(1-vinylimidazole) (PVIZ) was deposited via liquid-free initiated chemical vapor deposition (iCVD). Spectroscopy confirmed the identity of the polymer, which compared well to previous characterization studies of PVIZ deposited via liquid phase free radical polymerization. Kinetic studies found that the polymer deposition rate is first order with respect to the surface monomer concentration. Crosslinking of PVIZ was achieved by iCVD and the crosslinked polymer was integrated within a standard photoanode of a DSSC as a proof-of-concept device. The PVIZ DSSC using a conformal coating of the polymer achieved $0.80 \%$ efficiency, which was higher than the $0.63 \%$ efficiency without the polymer coating. The open circuit voltage and the short circuit current of the DSSC with the polymer electrolyte are higher than those of liquid electrolyte DSSCs. The increase in the voltage was attributed to a negative shift in the conduction band edge of the $\mathrm{TiO}_{2}$, while the increase in the current was ascribed to the polymer forming a 
blocking layer on the $\mathrm{TIO}_{2}$ surface, which helped reduce interfacial electron recombination with triiodide in the electrolyte.

\section{Acknowledgements}

The authors would like to thank the U.S. National Science Foundation (NSF) for support under Grant No. CBET-1236180. K.K.S.L. would also like to acknowledge support from the NSF under Grant No. CBET-0846245. This work made use of Drexel University's Centralized Research Facilities and lab facilities of Prof. Giuseppe Palmese and Prof. Caroline Schauer. We would also like to thank David Howe and Prof. Andrew Magenau for the GPC measurements.

\section{References}

(1) O'Regan, B. \& Grätzel, M. A low-cost, high-efficiency solar cell based on dyesensitized colloidal $\mathrm{TiO}_{2}$ films. Nature 353, 737-740, (1991).

(2) Kakiage, K., Aoyama, Y., Yano, T., Oya, K., Fujisawa, J.-i. \& Hanaya, M. Highlyefficient dye-sensitized solar cells with collaborative sensitization by silyl-anchor and carboxy-anchor dyes. Chemical Communications 51, 15894-15897, (2015).

(3) Baxter, J. B. Commercialization of dye sensitized solar cells: present status and future research needs to improve efficiency, stability, and manufacturing. Journal of Vacuum Science \& Technology A 30, 020801, (2012).

(4) Grätzel, M. Dye-sensitized solar cells. Journal of Photochemistry and Photobiology C: Photochemistry Reviews 4, 145-153, (2003).

(5) Nejati, S. \& Lau, K. K. Integration of polymer electrolytes in dye sensitized solar cells by initiated chemical vapor deposition. Thin Solid Films 519, 4551-4554, (2011).

(6) Smolin, Y. Y., Nejati, S., Bavarian, M., Lee, D., Lau, K. K. S. \& Soroush, M. Effects of polymer chemistry on polymer-electrolyte dye sensitized solar cell performance: A theoretical and experimental investigation. Journal of Power Sources 274, 156-164, (2015).

(7) Nejati, S. \& Lau, K. K. Pore filling of nanostructured electrodes in dye sensitized solar cells by initiated chemical vapor deposition. Nano Letters 11, 419-423, (2010).

(8) Li, B., Wang, L., Kang, B., Wang, P. \& Qiu, Y. Review of recent progress in solidstate dye-sensitized solar cells. Solar Energy Materials and Solar Cells 90, 549573, (2006). 
(9) Cai, N., Moon, S.-J., Cevey-Ha, L., Moehl, T., Humphry-Baker, R., Wang, P., Zakeeruddin, S. M. \& Grätzel, M. An organic D-m-A dye for record efficiency solid-state sensitized heterojunction solar cells. Nano Letters 11, 1452-1456, (2011).

(10) O'Regan, B., Lenzmann, F., Muis, R. \& Wienke, J. A solid-state dye-sensitized solar cell fabricated with pressure-treated $\mathrm{P} 25-\mathrm{TiO}_{2}$ and $\mathrm{CuSCN}$ : analysis of pore filling and IV characteristics. Chemistry of Materials 14, 5023-5029, (2002).

(11) Schmidt-Mende, L. \& Grätzel, M. TiO 2 pore-filling and its effect on the efficiency of solid-state dye-sensitized solar cells. Thin Solid Films 500, 296-301, (2006).

(12) Yum, J. H., Chen, P., Grätzel, M. \& Nazeeruddin, M. K. Recent developments in solid-state dye-sensitized solar cells. ChemSusChem 1, 699-707, (2008).

(13) Cho, W., Lim, J., Kim, T.-Y., Kim, Y. R., Song, D., Park, T., Fabregat-Santiago, F., Bisquert, J. \& Kang, Y. S. Electron-transfer kinetics through interfaces between electron-transport and ion-transport layers in solid-state dye-sensitized solar cells utilizing solid polymer electrolyte. Journal of Physical Chemistry C 120, 2494-2500, (2016).

(14) Song, D., Cho, W., Lee, J. H. \& Kang, Y. S. Toward higher energy conversion efficiency for solid polymer electrolyte dye-sensitized solar cells: ionic conductivity and $\mathrm{TiO}_{2}$ pore-filling. Journal of Physical Chemistry Letters 5, 12491258, (2014).

(15) Ng, H. M., Ramesh, S. \& Ramesh, K. Efficiency improvement by incorporating 1methyl-3-propylimidazolium iodide ionic liquid in gel polymer electrolytes for dyesensitized solar cells. Electrochimica Acta 175, 169-175, (2015).

(16) Huang, S. Y., Schlichthörl, G., Nozik, A. J., Grätzel, M. \& Frank, A. J. Charge recombination in dye-sensitized nanocrystalline $\mathrm{TiO}_{2}$ solar cells. Journal of Physical Chemistry B 101, 2576-2582, (1997).

(17) Su'ait, M., Rahman, M. \& Ahmad, A. Review on polymer electrolyte in dyesensitized solar cells (DSSCs). Solar Energy 115, 452-470, (2015).

(18) Pu, H., Qin, Y., Wan, D. \& Yang, Z. Proton-conducting polymers via free radical polymerization of diisopropyl-p-vinylbenzyl phosphonate and 1-vinylimidazole. Macromolecules 42, 3000-3004, (2009).

(19) Molina, M., Gómez-Antón, M., Rivas, B., Maturana, H. \& Pierola, I. Removal of $\mathrm{Hg}$ (II) from acid aqueous solutions by poly ( $\mathrm{N}$-vinylimidazole) hydrogel. Journal of Applied Polymer Science 79, 1467-1475, (2001).

(20) Lau, K. K. S. \& Gleason, K. K. Initiated chemical vapor deposition (iCVD) of poly(alkyl acrylates): a kinetic model. Macromolecules 39, 3695-3703, (2006).

(21) Lau, K. K. S. \& Gleason, K. K. Initiated chemical vapor deposition (iCVD) of poly(alkyl acrylates): an experimental study. Macromolecules 39, 3688-3694, (2006).

(22) Nazeeruddin, M. K., Kay, A., Rodicio, I., Humphry-Baker, R., Mueller, E., Liska, P., Vlachopoulos, N. \& Graetzel, M. Conversion of light to electricity by cis$\mathrm{X}_{2}$ bis(2,2'-bipyridyl-4,4'-dicarboxylate)ruthenium(II) charge-transfer sensitizers (X $=\mathrm{Cl}^{-}, \mathrm{Br}^{-}, \mathrm{I}^{-}, \mathrm{CN}^{-}$, and $\mathrm{SCN}^{-}$) on nanocrystalline titanium dioxide electrodes. Journal of the American Chemical Society 115, 6382-6390, (1993) 
(23) Talu, M., Demiroglu, E. U., Yurdakul, S. \& Badoglu, S. FTIR, Raman and NMR spectroscopic and DFT theoretical studies on poly( $N$-vinylimidazole). Spectrochimica Acta A 134, 267-275, (2015).

(24) Lippert, J., Robertson, J., Havens, J. R. \& Tan, J. S. Structural studies of poly (Nvinylimidazole) complexes by infrared and Raman spectroscopy. Macromolecules 18, 63-67, (1985).

(25) Gleason, K. K. CVD Polymers: Fabrication of Organic Surfaces and Devices. (John Wiley \& Sons, 2015).

(26) Yuan, S., Pehkonen, S. O., Liang, B., Ting, Y. P., Neoh, K. G. \& Kang, E. T. Poly(1-vinylimidazole) formation on copper surfaces via surface-initiated graft polymerization for corrosion protection. Corrosion Science 52, 1958-1968, (2010).

(27) Li, X., Goh, S. H., Lai, Y. H. \& Wee, A. T. S. Miscibility and interactions in blends of carboxyl-containing polysiloxane with poly(1-vinylimidazole). Polymer 42, 5463-5469, (2001).

(28) Odian, G. G. Principles of Polymerization. (Wiley, 1991).

(29) Reeja-Jayan, B., Kovacik, P., Yang, R., Sojoudi, H., Ugur, A., Kim, D. H., Petruczok, C. D., Wang, X., Liu, A. \& Gleason, K. K. A route towards sustainability through engineered polymeric interfaces. Advanced Materials Interfaces 1, 1400117 (2014).

(30) Bradley, L. C. \& Gupta, M. Copolymerization of 1-ethyl-3-vinylimidazolium bis(trifluoromethylsulfonyl)imide via initiated chemical vapor deposition. Macromolecules 47, 6657-6663, (2014).

(31) Kusama, H., Kurashige, M. \& Arakawa, H. Influence of nitrogen-containing heterocyclic additives in $\mathrm{I}^{-} / \mathrm{I}_{3}{ }^{-}$redox electrolytic solution on the performance of Ru-dye-sensitized nanocrystalline $\mathrm{TiO}_{2}$ solar cell. Journal of Photochemistry and Photobiology A 169, 169-176, (2005).

(32) Boschloo, G., Häggman, L. \& Hagfeldt, A. Quantification of the effect of 4-tertbutylpyridine addition to $\mathrm{I}^{-} / \mathrm{I}_{3}{ }^{-}$redox electrolytes in dye-sensitized nanostructured $\mathrm{TiO}_{2}$ solar cells. Journal of Physical Chemistry B 110, 13144-13150, (2006).

(33) Kalyanasundaram, K. Dye-Sensitized Solar Cells. (EPFL press, 2010).

(34) Kelly, C. A., Farzad, F., Thompson, D. W., Stipkala, J. M. \& Meyer, G. J. Cationcontrolled interfacial charge injection in sensitized nanocrystalline $\mathrm{TiO}_{2}$. Langmuir 15, 7047-7054, (1999).

(35) Pelet, S., Moser, J.-E. \& Grätzel, M. Cooperative effect of adsorbed cations and iodide on the interception of back electron transfer in the dye sensitization of nanocrystalline $\mathrm{TiO}_{2}$. Journal of Physical Chemistry B 104, 1791-1795, (2000).

(36) Pekel, N., Savaş, H. \& Güven, O. Complex formation and adsorption of $\mathrm{V}_{3}{ }^{+}, \mathrm{Cr}_{3}{ }^{+}$ and $\mathrm{Fe}_{3}{ }^{+}$ions with poly $(\mathrm{N}$-vinylimidazole). Colloid and Polymer Science 280, 4651, (2002).

(37) Rivas, B. L., Maturana, H. A., Molina, M. J., Gómez-Aantón, M. R. \& Piérola, I. F. Metal ion binding properties of poly ( $\mathrm{N}$-vinylimidazole) hydrogels. Journal of Applied Polymer Science 67, 1109-1118, (1998).

(38) Yu, H., Zhang, S., Zhao, H., Will, G. \& Liu, P. An efficient and low-cost $\mathrm{TiO}_{2}$ compact layer for performance improvement of dye-sensitized solar cells. Electrochimica Acta 54, 1319-1324, (2009). 
(39) Hore, S., Vetter, C., Kern, R., Smit, H. \& Hinsch, A. Influence of scattering layers on efficiency of dye-sensitized solar cells. Solar Energy Materials and Solar Cells 90, 1176-1188, (2006).

(40) Sommeling, P. M., O'Regan, B. C., Haswell, R. R., Smit, H. J. P., Bakker, N. J., Smits, J. J. T., Kroon, J. M. \& van Roosmalen, J. A. M. Influence of a $\mathrm{TiCl}_{4}$ posttreatment on nanocrystalline $\mathrm{TiO}_{2}$ films in dye-sensitized solar cells. Journal of Physical Chemistry B 110, 19191-19197, (2006). 
Table 1. FTIR major peak positions and assignments for VIZ and PVIZ.

\begin{tabular}{cl}
\hline $\begin{array}{c}\text { Peak Position } \\
\left(\mathbf{c m}^{-1}\right)\end{array}$ & Assignment \\
\hline 3108 & Imidazole ring $\mathrm{CH}$ stretch \\
$2935-2965$ & $\begin{array}{l}\text { Polymer backbone } \mathrm{CH} \text { and } \mathrm{CH}_{2} \text { stretch } \\
\text { (PVIZ only) }\end{array}$ \\
1647 & Vinyl C=C stretch (VIZ only) \\
1497 & Imidazole ring stretch \\
1284 & Imidazole ring CN stretch \\
1083 & Imidazole ring bend and stretch \\
744 & Imidazole ring bend \\
663 & Imidazole ring torsion \\
\hline
\end{tabular}


Table 2. Position and relative intensity of fitted N1s and C1s XPS peaks of iCVD PVIZ. Theoretical values are in parentheses.

\begin{tabular}{ccc|cccc}
\hline & \multicolumn{2}{c|}{ Nitrogen } & \multicolumn{3}{c}{ Carbon } \\
& $-\mathrm{N}<$ & $=\mathrm{N}-$ & $\mathrm{N}=\mathrm{C}-\mathrm{H}$ & $\mathbf{C}-\mathrm{N}$ & $\mathrm{C}-\mathrm{H}$ \\
\hline Position (eV) & 398.6 & 400.5 & 286.3 & 285.3 & 284.5 \\
\hline $\begin{array}{c}\text { Atomic \% } \\
\text { (theoretical) }\end{array}$ & 48.3 & 51.7 & 13.2 & 32.2 & 54.6 \\
$(50)$ & $(50)$ & $(11)$ & $(33)$ & $(55)$ \\
\hline
\end{tabular}




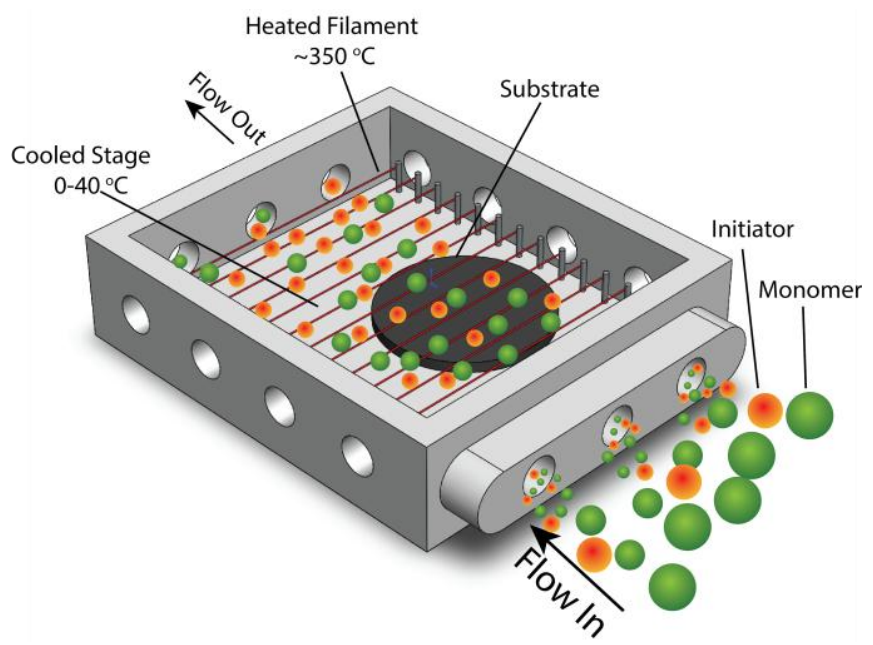

Figure 1. Schematic of the iCVD deposition process. Monomer (orange) and initiator (green) are introduced as vapors into the reaction chamber through three entry ports. The heated filaments are used to activate the initiator into free radicals. Monomer and activated initiator are absorbed onto the cooled substrate and surface polymerization occurs. 


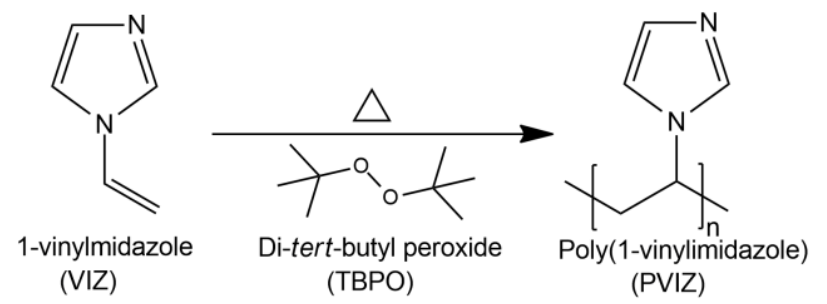

Figure 2. Free radical addition polymerization reaction of PVIZ. 


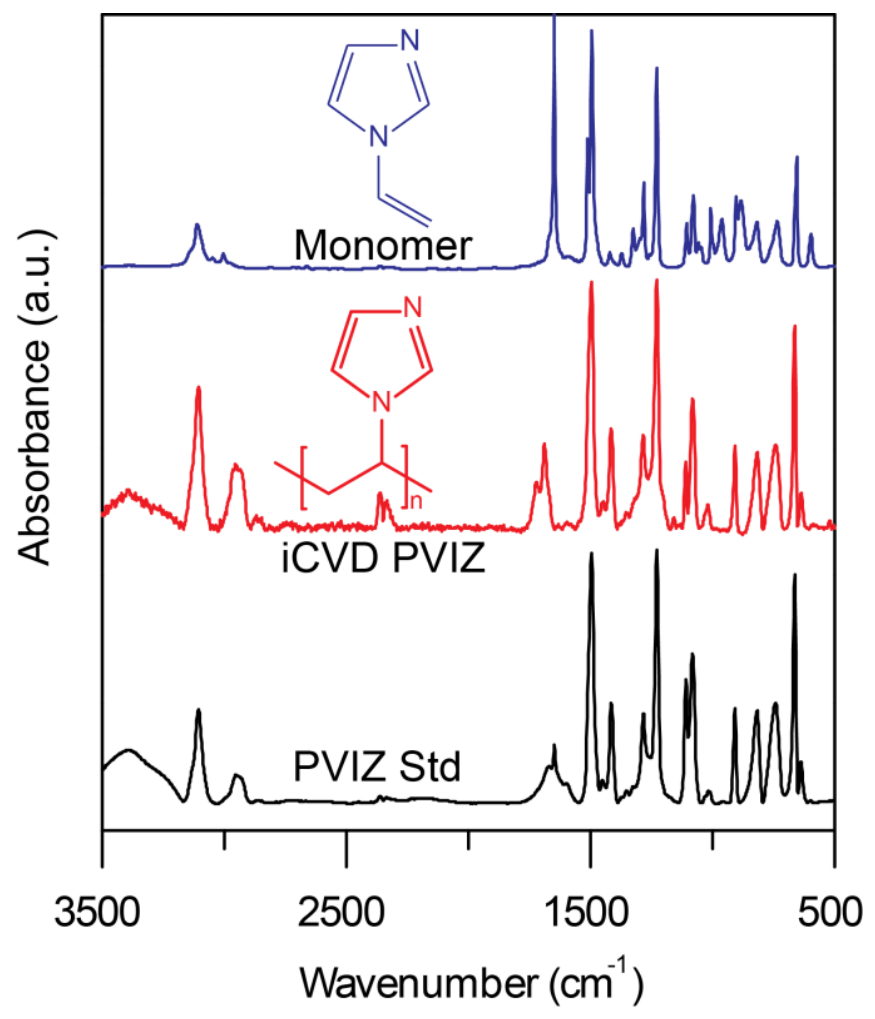

Figure 3. FTIR spectra of the VIZ monomer (top), iCVD PVIZ polymer (middle), and commercial PVIZ (bottom). 

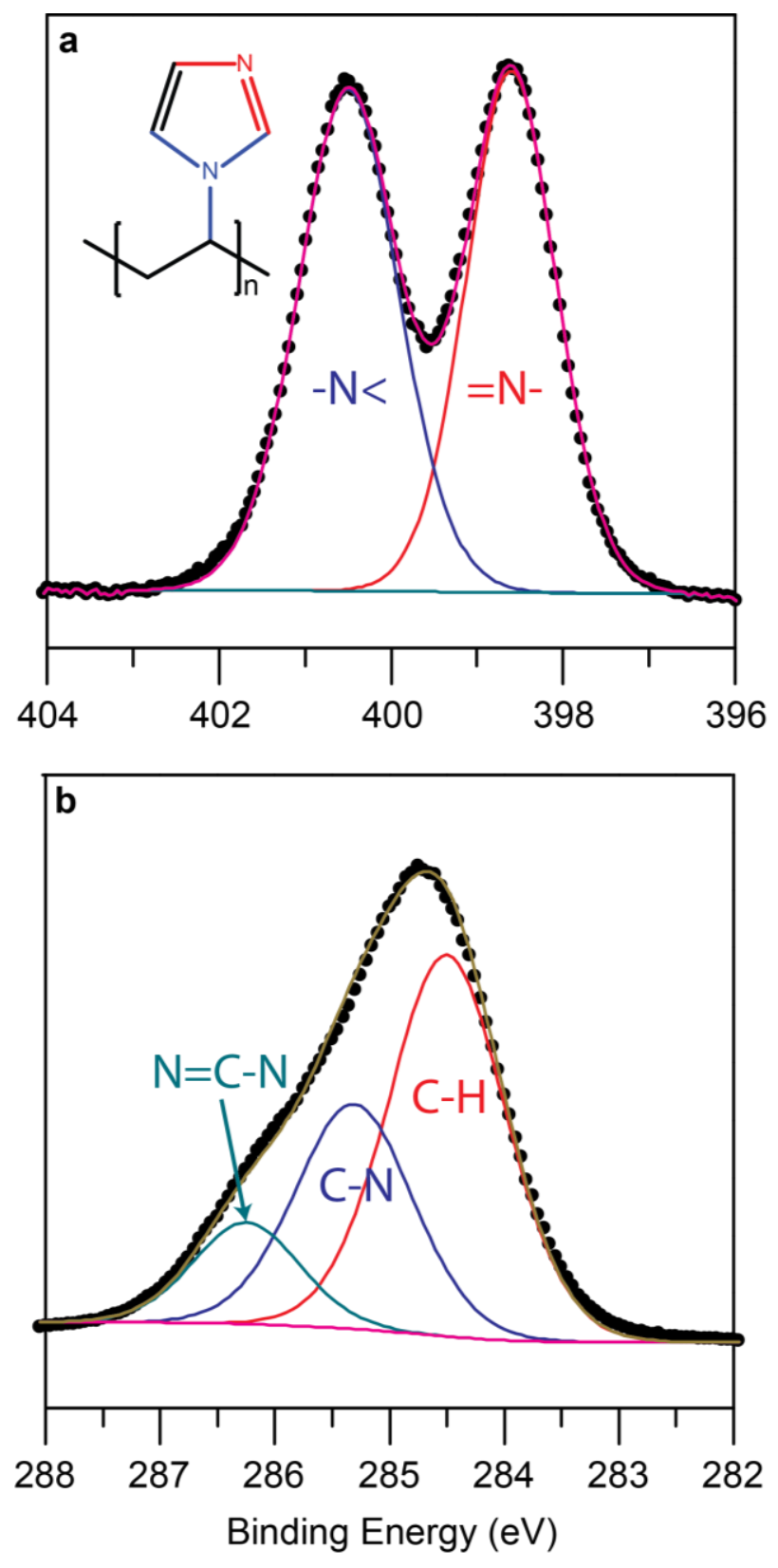

Figure 4. High resolution XPS spectra of (a) N1s and (b) C1s, and the corresponding fitted peaks of various bonding environments in each elemental region for iCVD PVIZ. 


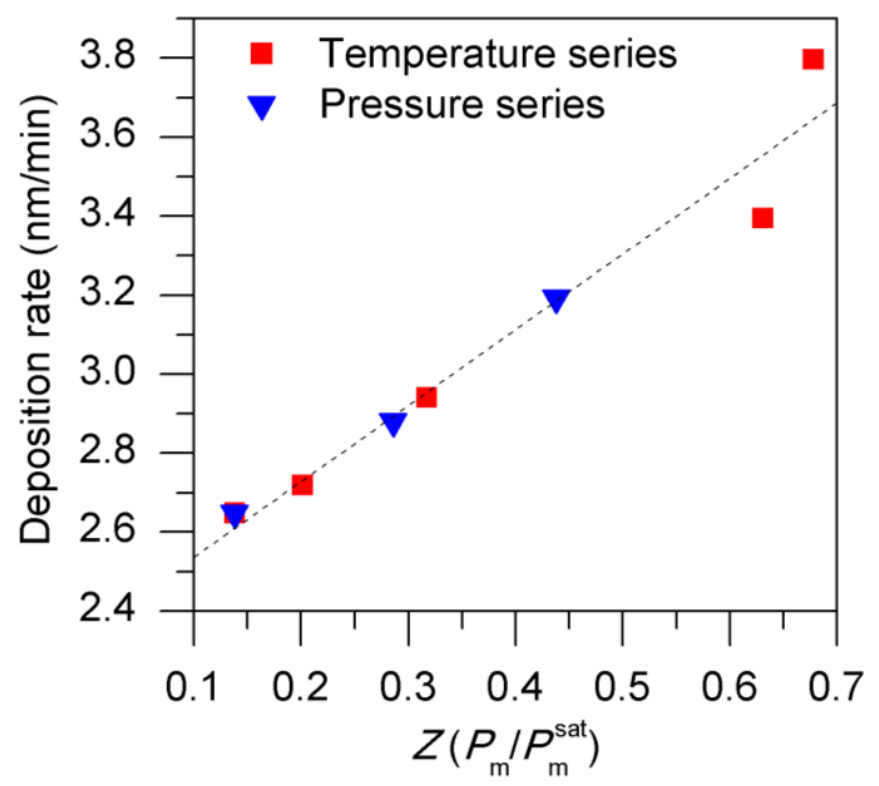

Figure 5. Deposition rate of iCVD PVIZ as a function of the fractional monomer saturation $Z$, which is a measure of the surface monomer concentration. 


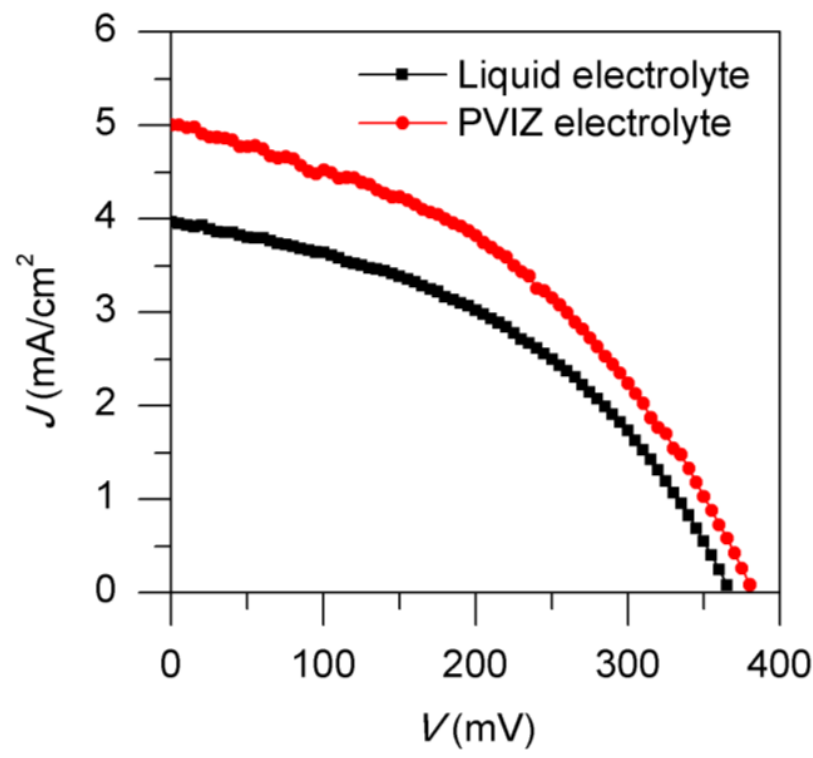

Figure 6. Photocurrent-voltage $(J-V)$ behavior of $\mathrm{PVIZ}$ and liquid electrolyte DSSCs. 


\section{Graphical Abstract}
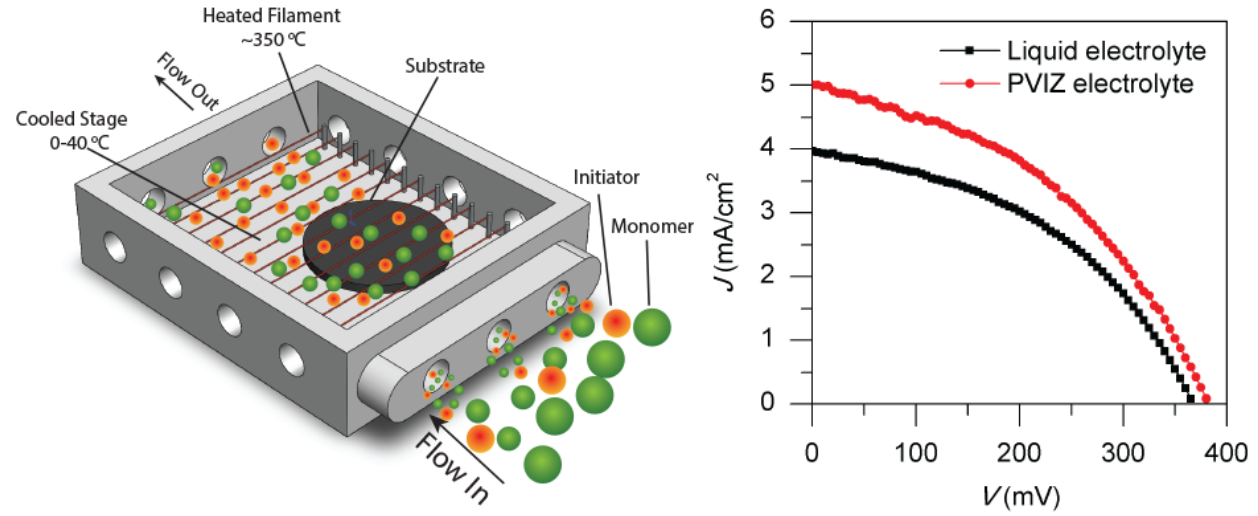